\title{
DIAMETER TWO PROPERTIES, CONVEXITY AND SMOOTHNESS
}

\author{
TROND A. ABRAHAMSEN, VEGARD LIMA, OLAV NYGAARD, \\ AND STANIMIR TROYANSKI
}

\begin{abstract}
We study smoothness and strict convexity of (the bidual) of Banach spaces in the presence of diameter 2 properties. We prove that the strong diameter 2 property prevents the bidual from being strictly convex and being smooth, and we initiate the investigation whether the same is true for the (local) diameter 2 property. We also give characterizations of the following property for a Banach space $X$ : "For every slice $S$ of $B_{X}$ and every norm-one element $x$ in $S$, there is a point $y \in S$ in distance as close to 2 as we want." Spaces with this property are shown to have non-smooth bidual.
\end{abstract}

\section{INTRODUCTION}

Let $X$ be a (real) Banach space and denote, as usual, by $B_{X}$ and $S_{X}$ its unit ball and unit sphere, respectively, and denote the topological dual of $X$ by $X^{*}$.

Recall that (the norm of) a Banach space $X$ is strictly convex if $\left\|\frac{x+y}{2}\right\|<1$ when $x$ and $y$ are different points of $S_{X}$, and that (the norm of) $X$ is smooth if for every $x \in S_{X}$ there is exactly one $x^{*} \in S_{X^{*}}$ such that $x^{*}(x)=1$. It is well-known that $X$ is smooth if $X^{*}$ is strictly convex, and that $X$ is strictly convex if $X^{*}$ is smooth.

It is a classical result from 1948 of J. Dixmier [10, Théorème 20'] that $X^{* * * *}$ is never strictly convex unless $X$ is reflexive. Several authors have independently strengthened Dixmier's result by showing that $X^{* * *}$ is not smooth for $X$ nonreflexive. (A partial list of authors can be found in [21. Milman credits the result to M. I. Kadets [18, Theorem 2.3].) Note that this result is sharp in the sense that James' space $J$ has a renorming such that the third dual is strictly convex 21.

The purpose of this paper is to study the implications of the big-slice phenomena on smoothness and convexity. By a slice of $B_{X}$ of $X$ we mean a set of the form

$$
S\left(x^{*}, \varepsilon\right):=\left\{x \in B_{X}: x^{*}(x)>1-\varepsilon, x^{*} \in S_{X^{*}}, \varepsilon>0\right\} .
$$

Recall the following successively stronger "big-slice concepts", defined in [4]:

Definition 1.1. A Banach space $X$ has the

(i) local diameter 2 property (LD2P) if every slice of $B_{X}$ has diameter 2.

2010 Mathematics Subject Classification. 46B04, 46B20.

Key words and phrases. Diameter 2 property; strictly convex norm; smooth norm; Daugavet property.

The main results of this paper were presented in a talk by the fourth named author at the Seminario Matematico e Fisico di Milano in March 2016. The fourth named author was partially supported by MTM2014-54182-P and the Bulgarian National Scientific Fund under Grant DFNI-I02/10. 
(ii) diameter 2 property (D2P) if every non-empty relatively weakly open subset of $B_{X}$ has diameter 2 .

(iii) strong diameter 2 property (SD2P) if every finite convex combination of slices of $B_{X}$ has diameter 2 .

In Section 2 we prove that $X^{* *}$ can be neither strictly convex nor smooth if $X$ has the SD2P. In fact, we prove that when $X$ has the SD2P, then $X^{* *}$ contains an isometric copy of $L_{1}[0,1]$. We next ask whether it is possible that $X$ can have (L)D2P while $X^{* *}$ is still strictly convex, and we give a partial answer; namely we prove that if $X$ has a bimonotone basis and the $\mathrm{D} 2 \mathrm{P}$, then the unit sphere of $X^{* *}$ contains a line segment of length as close to 1 as we want.

Recall the following successively stronger "rotundity concepts":

Definition 1.2. A Banach space $X$ is

(i) strictly convex (or rotund) if every $x \in S_{X}$ is an extreme point in $B_{X}$, i.e., for every $y \in X$ we have that $y=0$ whenever $\|x \pm y\|=1$.

(ii) weakly midpoint locally uniformly rotund (weakly MLUR) if every $x \in S_{X}$ is a weakly strongly extreme point of $B_{X}$, i.e., for every sequence $\left(x_{n}\right)$ in $X$, we have that $x_{n} \rightarrow 0$ weakly whenever $\left\|x \pm x_{n}\right\| \rightarrow 1$.

(iii) midpoint locally uniformly rotund (MLUR) if every $x \in S_{X}$ is a strongly extreme point of $B_{X}$, i.e., for every sequence $\left(x_{n}\right)$ in $X$, we have that $x_{n} \rightarrow 0$ in norm whenever $\left\|x \pm x_{n}\right\| \rightarrow 1$.

It is clear that if $X$ is weakly MLUR then $X$ is strictly convex. Smith [22] observed using the Principle of Local Reflexivity that $X$ is weakly MLUR if and only if every $x \in S_{X}$ is an extreme point of $B_{X^{* *}}$. In particular, if $X^{* *}$ is strictly convex, then $X$ is weakly MLUR. The converse is not true.

It was observed in [2, Proposition 1.3] that if $X$ is weakly MLUR, then the LD2P implies the D2P by Choquet's lemma [11, Lemma 3.69]. In particular, the LD2P implies the D2P when $X^{* *}$ is strictly convex.

The main result of [2] is that there exists an equivalent norm $|\cdot|$ on $C[0,1]$ such that $X=(C[0,1],|\cdot|)$ is MLUR and has the $(\mathrm{L}) \mathrm{D} 2 \mathrm{P}$. In fact $X$ has the $\mathrm{LD} 2 \mathrm{P}$ in the following stronger sense:

Definition 1.3. A Banach space $X$ has the local diameter 2 property+ $(L D 2 P+)$ if for every $\varepsilon>0$, every slice $S$ of $B_{X}$, and every $x \in S \cap S_{X}$ there exists $y \in S$ such that $\|x-y\|>2-\varepsilon$.

Let $X$ be a Banach space and $I$ the identity operator on $X$. Recall that $X$ has the Daugavet property if the equation

$$
\|I+T\|=1+\|T\|
$$

holds for every rank 1 operator $T$ on $X$. The Daugavet property can be characterized as follows (see [24] or [20]):

Theorem 1.4. Let $X$ be a Banach space. Then the following statements are equivalent.

(i) $X$ has the Daugavet property.

(ii) The equation $\|I+T\|=1+\|T\|$ holds for every weakly compact operator $T$ on $X$.

(iii) For every $\varepsilon>0$, every $x \in S_{X}$, and every $x^{*} \in S_{X^{*}}$, there exists $y \in$ $S\left(x^{*}, \varepsilon\right)$ such that $\|x+y\| \geq 2-\varepsilon$. 
(iv) For every $\varepsilon>0$, every $x^{*} \in S_{X^{*}}$, and every $x \in S_{X}$, there exists $y^{*} \in$ $S(x, \varepsilon)$ such that $\left\|x^{*}+y^{*}\right\| \geq 2-\varepsilon$.

(v) For every $\varepsilon>0$ and every $x \in S_{X}$ we have $B_{X}=\overline{c o n v}\left(\Delta_{\varepsilon}(x)\right)$, where $\Delta_{\varepsilon}(x)=\left\{y \in B_{X}:\|y-x\| \geq 2-\varepsilon\right\}$.

In Section 3 we prove a similar characterization of the $\mathrm{LD} 2 \mathrm{P}+$, see Theorems 3.2 and 3.5. It is known that the dual of a Banach space with the Daugavet property is neither strictly convex nor smooth [17, Corollary 2.13]. In Corollary 3.7 we show that if $X$ has the $\mathrm{LD} 2 \mathrm{P}+$, then $X^{* *}$ is not smooth. We also prove that just like the diameter two properties above the LD2P + is inherited by ai-ideals (we postpone the definition of this concept till we need it).

The notation and conventions we use are standard and follow [16. When considered necessary, notation and concepts are explained as the text proceeds.

\section{Strict CONVEXity And Smoothness of $X^{* *}$}

A result of Day [8, Theorem 9] says that neither $\ell_{1}(\Gamma), \Gamma$ uncountable, nor $\ell_{\infty}$ have equivalent smooth renormings. So for example no equivalent norm on $C[0,1]$ has a bidual that is smooth or strictly convex.

Our first aim in this section is to prove that if a Banach space $X$ has the SD2P, then the bidual is neither smooth nor strictly convex. Banach spaces which are M-ideals in their biduals are called $M$-embedded. It is known that non-reflexive M-embedded spaces have the SD2P [4, Theorem 4.10]. From [7, p. 109] (see also [14, Proposition I.1.7]) it is clear that the bidual of a non-reflexive M-embedded space is neither smooth nor strictly convex.

Let us note that in general we cannot say anything about the absence of smoothness or convexity in $X$ and $X^{*}$ in the presence of the SD2P. Indeed, there exists a smooth M-embedded renorming $X$ of $c_{0}$ with strictly convex dual [14, Corollary III.2.12]. There also exists a strictly convex M-embedded space $X$ with smooth dual [14, Remark IV.1.17].

We will need the following concept:

Definition 2.1. A sequence $\left(x_{n}\right)$ in $X$ is said to be asymptotically isometric $\ell_{1}$, if there exists a sequence $\left(\delta_{n}\right)$ in $(0,1)$ decreasing to 0 and such that

$$
\sum_{n=1}^{m}\left(1-\delta_{n}\right)\left|a_{n}\right| \leq\left\|\sum_{n=1}^{m} a_{n} x_{n}\right\| \leq \sum_{n=1}^{m}\left|a_{n}\right|
$$

for each finite sequence $\left(a_{n}\right)_{n=1}^{m}$ in $\mathbb{R}$.

From [12, Remark II.5.2] we have the following:

Definition 2.2. A Banach space $X$ is said to be octahedral if for every finite dimensional subspace $E$ of $X$ and every $\varepsilon>0$, there exists $y \in S_{X}$ such that for every $x \in E$ and every $\lambda \in \mathbb{R}$, we have

$$
\|x+\lambda y\| \geq(1-\varepsilon)(\|x\|+|\lambda|) .
$$

Lemma 2.3. If $X$ is octahedral, then $X$ contains an asymptotically isometric $\ell_{1}$ sequence.

The proof uses an idea of H. Pfitzner (see [19, Theorem 2]). 
Proof. Let $\left(\delta_{n}\right) \subset(0,1)$ such that $\delta_{n} \rightarrow 0$. Let $\eta_{1}=\frac{\delta_{1}}{2}$ and $\eta_{n+1}=\frac{1}{2} \min \left\{\eta_{n}, \delta_{n+1}\right\}$. We will find a sequence $\left(x_{n}\right) \subset S_{X}$ such that

$$
\sum_{n=1}^{m}\left(1-\delta_{n}\right)\left|a_{n}\right|+\eta_{m} \sum_{n=1}^{m}\left|a_{n}\right| \leq\left\|\sum_{n=1}^{m} a_{n} x_{n}\right\|
$$

by induction. The $m=1$ step is trivial.

Assume (1) holds for a fixed $m \geq 1$. Choose $\varepsilon>0$ such that

$$
\varepsilon \leq \frac{\eta_{m}-\eta_{m+1}}{1-\delta_{n}+\eta_{m}}
$$

for $n=1,2, \ldots, m$ and

$$
\varepsilon \leq \delta_{m+1}-\eta_{m+1}
$$

Find, using the assumption and octahedrality, $x_{m+1} \in S_{X}$ such

$$
(1-\varepsilon)\left(\sum_{n=1}^{m}\left(1-\delta_{n}\right)\left|a_{n}\right|+\eta_{m} \sum_{n=1}^{m}\left|a_{n}\right|+\left|a_{m+1}\right|\right) \leq\left\|\sum_{n=1}^{m+1} a_{n} x_{n}\right\| .
$$

Then

$$
\sum_{n=1}^{m+1}\left(1-\delta_{n}\right)\left|a_{n}\right|+\eta_{m+1} \sum_{n=1}^{m+1}\left|a_{n}\right| \leq\left\|\sum_{n=1}^{m+1} a_{n} x_{n}\right\|
$$

because the left hand side of (2) in this case will be greater than the left hand side of (3).

Remark 2.4. As noted above there is a smooth M-embedded Banach space $X$ with strictly convex dual and a strictly convex M-embedded Banach space $X$ with smooth dual. By [19, Theorem 2] $X^{*}$ contains an asymptotically isometric $\ell_{1}$ sequence whenever $X$ is M-embedded. Hence the presence of an asymptotically isometric $\ell_{1}$ sequence in a Banach space $X$ does not prevent $X$ from being strictly convex or smooth - even when $X$ is a dual space.

Theorem 2.5. If $X$ has the SD2P, then $X^{* *}$ contains an isometric copy of $L_{1}[0,1]$.

Proof. We know from [13, Theorem 2.4] that $X$ has the SD2P if and only if $X^{*}$ is octahedral. From Lemma 2.3 we know that an octahedral space contains an asymptotically isometric $\ell_{1}$ sequence. From [9, Theorem 2] we then have that $X^{* *}$ contains an isometric copy of $L_{1}[0,1]$.

Since $L_{1}[0,1]$ is neither smooth nor strictly convex the following corollary is immediate.

Corollary 2.6. If $X$ has the SD2P, then $X^{* *}$ is neither strictly convex nor smooth.

From Corollary 2.6 a natural question arises: If $X$ has the D2P, can $X^{* *}$ be strictly convex? (Recall from the Introduction that when $X^{* *}$ is strictly convex, LD2P and D2P for $X$ must be the same thing.) We will give a negative answer to this question in the case $X$ has a bimonotone basis in Proposition 2.10 below.

We start with an alternative description of the D2P.

Proposition 2.7. The following statements are equivalent: 
(i) X has the D2P.

(ii) Whenever $\varepsilon>0, x \in X$ with $\|x\|<1$, and $F$ is a finite dimensional subspace of $X^{*}$, there exist $y_{1}, y_{2} \in F_{\perp}$ with $\left\|x+y_{i}\right\|<1, i=1,2$, such that $\left\|y_{1}-y_{2}\right\|>2-\varepsilon$.

(iii) Whenever $\varepsilon>0, x \in X$ with $\|x\|<1$, and $E$ is a finite co-dimensional subspace of $X$, there exists $y_{1}, y_{2} \in E$ with $\left\|x+y_{i}\right\|<1, i=1,2$, such that $\left\|y_{1}-y_{2}\right\|>2-\varepsilon$.

Proof. (i) $\Rightarrow$ (iii). Let $\varepsilon>0, x \in X$ with $\|x\|<1$, and $E$ a finite co-dimensional subspace of $X$. Assume without loss of generality that $E$ does not contain $x$. Choose a finite dimensional subspace $F$ of $X$ which contains $x$ and with the property that $X=E \oplus F$. Let $P$ be a bounded linear projection onto $F$. For $\varepsilon / 5>\delta>0$ put

$$
W=\left\{w \in B_{X}:\|P(x-w)\|<\delta\right\} .
$$

Note that $W$ is a neighbourhood of $x$ in the relative weak topology on $B_{X}$. Now, using (i), and that non-empty relatively weakly open subsets of $B_{X}$ has diameter 2 , we may pick $w_{1}, w_{2}$ in $W$, both of norm $<1-\delta$ and with $\left\|w_{2}-w_{1}\right\|>2-3 \delta$. have

Put $y_{i}=w_{i}-P w_{i}$. Then $y_{1}$ and $y_{2}$ are both in $E$. Moreover, for $i=1,2$, we

We also have

$$
\left\|x+y_{i}\right\|=\left\|P x+w_{i}-P w_{i}\right\| \leq\left\|P\left(x-w_{i}\right)\right\|+\left\|w_{i}\right\|<1 .
$$

$$
\begin{aligned}
\left\|y_{1}-y_{2}\right\| & =\left\|w_{1}-w_{2}-P\left(w_{1}-w_{2}\right)\right\| \\
& \geq\left\|w_{1}-w_{2}\right\|-2 \delta>2-5 \delta>2-\varepsilon
\end{aligned}
$$

since $\left\|P\left(w_{1}-w_{2}\right)\right\|<2 \delta$.

(iii) $\Rightarrow$ (ii). This is obvious as any finite dimensional subspace of a dual space has a finite co-dimensional pre-annihilator.

(ii) $\Rightarrow$ (i). Let $\varepsilon>0$ and $U$ a non-empty relatively weakly open subset of $B_{X}$. Let $x \in U$ with $\|x\|<1$ and find a set of the form

$$
V=\left(x+\bigcap_{k=1}^{n}\left(f_{k}\right)^{-1}(-\delta, \delta)\right) \bigcap B_{X} \subset U,
$$

where $\left(f_{k}\right)_{k=1}^{n} \subset S_{X^{*}}$ and $\delta>0$. Let

$$
F=\operatorname{span}\left\{\left(f_{k}\right)_{k=1}^{n}\right\} .
$$

As $F$ is of finite dimension in $X^{*}$, there exist $y_{1}, y_{2} \in F_{\perp}$ with $\left\|x+y_{i}\right\|<1$, $i=1,2$, such that $\left\|y_{2}-y_{1}\right\|>2-\varepsilon$. For $i=1,2$ we have $x+y_{i} \in V$ with $\left\|\left(x+y_{1}\right)-\left(x+y_{2}\right)\right\|>2-\varepsilon$.

As a first application of Proposition 2.7 let us give a very simple proof of the following fact, known from [6].

Proposition 2.8. If $X$ has the D2P and $Y$ is a subspace of $X$ with finite codimension, then $Y$ has the D2P.

Proof. If $y \in Y$ with $\|y\|<1, \varepsilon>0$, and $E$ is of finite co-dimension in $Y$, then $E$ is also of finite co-dimension in $X$ and the result follows from Proposition 2.7(iii).

Now we return to the problem whether $X^{* *}$ can be strictly convex if $X$ has the $\mathrm{D} 2 \mathrm{P}$. 
Definition 2.9. A Schauder basis $\left(e_{k}\right)_{k=1}^{\infty}$ for a Banach space $X$ is bimonotone if the projections

$$
P_{[n, m]}\left(\sum_{k=1}^{\infty} a_{k} e_{k}\right)=\sum_{k=n}^{m} a_{k} e_{k} .
$$

satisfy $\left\|P_{[n, m]}\right\|=1$ if $n \leq m$.

Proposition 2.10. Suppose $X$ has a bimonotone basis. Then, if $X$ has the D2P and $\varepsilon>0, S_{X^{* *}}$ contains a line segment of length $>1-\varepsilon$.

Proof. Let $P_{n}$ be the natural projections associated to the basis $\left(e_{i}\right)$ and put $Q_{n}=I-P_{n}$. Let $\varepsilon>0$ and define $\varepsilon_{n}=\varepsilon / 2^{n+1}$.

Define $s_{1}=x_{1}=\frac{\left(1-\varepsilon_{1}\right) e_{1}}{\left\|e_{1}\right\|}$. Assume that we have found a sequence $\left(x_{i}\right)_{i=1}^{k}$ each with finite support $\operatorname{supp}\left(x_{i}\right)=\left[l_{i}, r_{i}\right]$ such that if $s_{k}=\sum_{i=1}^{k} x_{i}$, then

- $\left\|s_{k}\right\|<1$ and $\left\|s_{k}\right\| \geq\left\|s_{k-1}\right\|$

- $\left\|x_{i}\right\|>1-\varepsilon_{i}$ for $i=1,2, \ldots, k$.

- $r_{i}<l_{i+1}$ for $i=1,2, \ldots, k-1$.

Let us show how to find $x_{k+1}$. Let $E=Q_{r_{k}}(X)$ and use Proposition 2.7 to find $y_{1}, y_{2} \in E$ with $\left\|s_{k}+y_{i}\right\|<1$ and $\left\|y_{1}-y_{2}\right\|>2-2 \varepsilon_{k+1}$. Without loss of generality $\left\|y_{1}\right\| \geq\left\|y_{2}\right\|$ and $y_{1}$ has finite support. Let $x_{k+1}=y_{1}$. Then $\left\|s_{k+1}\right\|=\left\|s_{k}+x_{k+1}\right\|<1$ and $\left\|s_{k}\right\| \leq\left\|s_{k+1}\right\|$ since the basis is monotone. We also have

$$
2\left\|x_{k+1}\right\|=2\left\|y_{1}\right\| \geq\left\|y_{1}-y_{2}\right\|>2-2 \varepsilon_{k+1}
$$

so $\left\|x_{k+1}\right\|>1-\varepsilon_{k+1}$.

Let $\mathcal{U}$ be a non-trivial ultrafilter on $\mathbb{N}$. Then $z=w^{*}-\lim _{\mathcal{U}} s_{m} \in X^{* *}$ exists with $\|z\| \leq 1$. For $\lambda \in[0,1]$, let

$$
z_{\lambda}=w^{*}-\lim _{\mathcal{U}}\left(s_{m}-\lambda s_{1}\right)=z-\lambda s_{1} .
$$

We have $z_{\lambda}=\lambda z_{1}+(1-\lambda) z_{0}$. Note that $\left\|s_{m}-s_{1}\right\| \leq\left\|s_{m}\right\|<1$ since the basis is bimonotone. Hence $\left\|z_{1}\right\| \leq 1$ and $\left\|z_{0}\right\| \leq 1$, so the line segment $\left[z_{0}, z_{1}\right]$ is contained in $B_{X^{* * *}}$. Let $R_{n}=P_{\left[l_{n}, r_{n}\right]}$ be the projection onto the support of $x_{n}$. We have $\left\|R_{n}\right\|=1$ and

$$
R_{n}^{* *} z_{\lambda}=w^{*}-\lim _{\mathcal{U}} R_{n}\left(s_{m}-\lambda s_{1}\right)=x_{n}
$$

and hence $\left\|z_{\lambda}\right\| \geq\left\|R_{n}^{* *} z_{\lambda}\right\|=\left\|x_{n}\right\|>1-\varepsilon_{n}$ for all $n$ which means that $\left\|z_{\lambda}\right\|=1$. Thus $z_{\lambda}=\lambda z_{1}+(1-\lambda) z_{0}, \lambda \in[0,1]$, is a line segment on the sphere. The segment has length $\left\|z_{0}-z_{1}\right\|=\left\|s_{1}\right\|>1-\varepsilon$.

\section{The LOCAL DiAmeter 2 PROPERTY +}

Let us recall from the Introduction the definition of the LD2P+.

Definition 3.1. We say that a Banach space $X$ has the local diameter 2 property $+(\mathrm{LD} 2 \mathrm{P}+)$ if for every $x^{*} \in S_{X^{*}}$, every $\varepsilon>0$, every $\delta>0$, and every $x \in S\left(x^{*}, \varepsilon\right) \cap S_{X}$ there exists $y \in S\left(x^{*}, \varepsilon\right)$ with $\|x-y\|>2-\delta$.

From [15, Theorem 1.4] and [24, Open problem (7) p. 95] the following is known.

Theorem 3.2. Let $X$ be a Banach space. Then the following statements are equivalent. 
(i) The equation $\|I-P\|=2$ holds for every norm 1 rank 1 projection $P$ on $X$.

(ii) For every $\varepsilon>0$, every $x^{*} \in S_{X^{*}}$ and every $x \in S\left(x^{*}, \varepsilon\right) \cap S_{X}$ there exists $y \in S\left(x^{*}, \varepsilon\right)$ with $\|x-y\|>2-\varepsilon$.

(iii) For every $x \in S_{X}$ and every $\varepsilon>0$ we have $x \in \overline{\operatorname{conv}}\left(\Delta_{\varepsilon}(x)\right)$, where $\Delta_{\varepsilon}(x)=\left\{y \in B_{X}:\|x-y\|>2-\varepsilon\right\}$.

From Lemma 3.3 below, which is due to Ivakhno and Kadets [15, Lemma 2.1], it is clear that the LD2P + is equivalent to the statements in Theorem 3.2. Therefore every Daugavet space has the LD2P+. Note, however, that the converse is not true as the LD2P + is stable by taking unconditional sums of Banach spaces which fails for spaces with the Daugavet property (see e.g. [15, Corollary 3.1]).

Lemma 3.3 (Ivakhno and Kadets). Let $\varepsilon>0$ and $x^{*} \in S_{X^{*}}$. Then for every $x \in S\left(x^{*}, \varepsilon\right) \cap S_{X}$ and every positive $\delta<\varepsilon$ there exist $y^{*} \in S_{X^{*}}$ such that $x \in S\left(y^{*}, \delta\right)$ and $S\left(y^{*}, \delta\right) \subset S\left(x^{*}, \varepsilon\right)$.

In the proof of Theorem 3.5 below we will need the following weak*-version of Lemma 3.3. Its proof is more or less verbatim to that of Lemma 3.3 and will therefore be omitted.

Lemma 3.4. Let $\varepsilon>0$ and $x \in S_{X}$. Then for every $x^{*} \in S(x, \varepsilon) \cap S_{X^{*}}$ which attains its norm and every positive $\delta<\varepsilon$ there exist $y \in S_{X}$ such that $x^{*} \in S(y, \delta)$ and $S(y, \delta) \subset S(x, \varepsilon)$.

We will now add to the list of statements in Theorem 3.2 statements similar to (ii) and (iv) in Theorem 1.4. As pointed out in [2, p. 232] the equivalence of (i) and (ii) in Theorem 3.5 below can be proved by a similar argument to the proof of [17, Lemma 1.5].

Theorem 3.5. Let $X$ be a Banach space. Then the following statements are equivalent:

(i) $X$ has the LDQP+.

(ii) For every $x \in S_{X}$, every $\varepsilon>0$, every $\delta>0$, and every $x^{*} \in S(x, \varepsilon) \cap S_{X^{*}}$ there exists $y^{*} \in S(x, \varepsilon)$ with $\left\|x^{*}-y^{*}\right\|>2-\delta$.

(iii) The equation $\|I-P\|=1+\|P\|$ holds for every weakly compact projection $P$ on $X$.

Proof. (i) $\Rightarrow$ (ii). By the Bishop-Phelps theorem we can assume without loss of generality that $x^{*} \in S(x, \varepsilon) \cap S_{X^{*}}$ attains its norm. Let $0<\eta<\min \{\varepsilon, \delta / 2\}$ and find by Lemma $3.4 y \in S_{X}$ such that $x^{*} \in S(y, \eta)$ and $S(y, \eta) \subset S(x, \varepsilon)$. Note that $y \in S\left(x^{*}, \eta\right)$ and thus, since $X$ has the LD2P+, we can find $z \in S\left(x^{*}, \eta\right)$ such that $\|y-z\|>2-\eta$. Hence there is $y^{*} \in S_{X^{*}}$ such that

$$
y\left(y^{*}\right)-z\left(y^{*}\right)=(y-z)\left(y^{*}\right)>2-\eta .
$$

From this we have $y\left(y^{*}\right)>1-\eta$ and $-z\left(y^{*}\right)>1-\eta$. It follows that $y^{*} \in S(x, \varepsilon)$ as $S(y, \eta) \subset S(x, \varepsilon)$. Moreover, using that $z \in S\left(x^{*}, \eta\right)$, we have

$$
\begin{aligned}
\left\|x^{*}-y^{*}\right\| & \geq\left(x^{*}-y^{*}\right)(z) \\
& =x^{*}(z)-y^{*}(z) \\
& >1-\eta+1-\eta>2-\delta .
\end{aligned}
$$


(ii) $\Rightarrow$ (i). The proof is identical to the proof of the converse except that one does not use the Bishop-Phelps theorem and that one uses Lemma 3.3 in place of Lemma 3.4

(i) $\Rightarrow$ (iii), The proof is similar to that of [17, Theorem 2.3].

(iii) $\Rightarrow$ (i). This is clear as (iii) trivially implies (i) in Theorem 3.2 .

Note that from Theorem 3.5 we get

Corollary 3.6. If $B_{X^{*}}$ contains a weak ${ }^{*}$-denting point, in particular if $X^{*}$ has the $R N P$, then $X$ does not have the LDQP+.

It is known that if $X^{* *}$ is smooth, then $X^{*}$ has the RNP (see e.g. [23]), hence we have the following corollary.

Corollary 3.7. If $X$ has the $L D 2 P+$, then $X^{* *}$ is not smooth.

It is known that all the diameter 2 properties in Definition 1.1 as well as the Daugavet property are inherited by certain subspaces called ai-ideals (see [5] and 11). We will end this section by showing that this is true for the LD2P+ as well.

A subspace $X$ of a Banach space $Y$ is called an ideal in $Y$ if there exists a norm 1 projection $P$ on $Y^{*}$ with $\operatorname{ker} P=X^{\perp}$. $X$ being an ideal in $Y$ is in turn equivalent to $X$ being locally 1-complemented in $Y$, i.e., for every $\varepsilon>0$ and every finite dimensional subspace $E \subset Y$ there exists a linear $T: E \rightarrow X$ such that

(i) $T e=e$ for all $e \in X \cap E$.

(ii) $\|T e\| \leq(1+\varepsilon)\|e\|$ for all $e \in E$.

Following [5] a subspace $X$ of a Banach space $Y$ is called an almost isometric ideal (ai-ideal) in $Y$ if $X$ is locally 1-complemented with almost isometric local projections, i.e., for every $\varepsilon>0$ and every finite-dimensional subspace $E \subset Y$ there exists $T: E \rightarrow X$ which satisfies a) and

(ii') $(1-\varepsilon)\|e\| \leq\|T e\| \leq(1+\varepsilon)\|e\|$ for all $e \in E$.

Note that an ideal $X$ in $Y$ is an ai-ideal if $P\left(Y^{*}\right)$ is a 1-norming subspace of $Y^{*}$ 5, Proposition 2.1]. Ideals $X$ in $Y$ for which $P\left(Y^{*}\right)$ is a 1-norming subspace for $Y$ are called strict ideals. An ai-ideal is, however, not necessarily strict (see [5, Example 1] and [3, Remark 3.2]).

Proposition 3.8. Let $Y$ have the LD2P+ and assume $X$ is an ai-ideal in $Y$. Then $X$ has the LD2P+.

Proof. For $\delta>0, Z$ a subspace of $Y$, and $x \in S_{Z}$ put

$$
\Delta_{\delta}^{Z}(x)=\left\{y \in B_{Z}:\|x-y\|>2-\delta\right\} .
$$

Let $x \in S_{X}, \varepsilon>0$, and $\alpha>0$. We will show that there exists $z \in \operatorname{conv} \Delta_{\varepsilon}^{X}(x)$ with $\|x-z\|<\alpha$. The result will then follow from Theorem 3.2 (iii). First, since $Y$ enjoys the LD2P+, we know that for any positive $\beta<\varepsilon$ and any positive $\gamma<\alpha$ we can find $y=\sum_{n=1}^{N} \lambda_{n} y_{n}$ with $\left(y_{n}\right)_{n=1}^{N} \subset \Delta_{\beta}^{Y}(x)$ such that $\|x-y\|<\gamma$. Now let $E=\operatorname{span}\left\{y_{1}, \ldots, y_{N}, x\right\}$ and pick a local projection $T: E \rightarrow X$ such that $T$ is a $(1+\eta)$-isometry with $\eta>0$ so small that $(1+\eta) \gamma+\eta<\alpha$, and $(1-\eta)(2-\beta)-\eta>2-\varepsilon$. Put $z_{n}=\frac{T y_{n}}{\left\|T y_{n}\right\|}$ and $z=\sum_{n=1}^{N} \lambda_{n} z_{n}$. As $T x=x$ we 
get

$$
\begin{aligned}
\|x-z\| & \leq\|x-T y\|+\|T y-z\| \\
& \leq\|T(x-y)\|+\sum_{n=1}^{N} \lambda_{n}\left|1-\left\|T y_{n}\right\|\right| \\
& <(1+\eta) \gamma+\max _{1 \leq n \leq N}\left|1-\left\|T y_{n}\right\|\right| \\
& \leq(1+\eta) \gamma+\eta<\alpha .
\end{aligned}
$$

Moreover, for every $1 \leq n \leq N$ we have,

$$
\begin{aligned}
\left\|x-z_{n}\right\| & =\left\|T\left(x-\frac{y_{n}}{\left\|T y_{n}\right\|}\right)\right\| \\
& \geq(1-\eta)\left\|x-\frac{y_{n}}{\left\|T y_{n}\right\|}\right\| \\
& \geq(1-\eta)\left(\left\|x-y_{n}\right\|-\left\|y_{n}-\frac{y_{n}}{\left\|T y_{n}\right\|}\right\|\right) \\
& \geq(1-\eta)\left(2-\beta-\frac{\left|1-\left\|T y_{n}\right\|\right|}{\left\|T y_{n}\right\|}\left\|y_{n}\right\|\right) \\
& \geq(1-\eta)\left(2-\beta-\frac{\eta}{1-\eta}\right)>2-\varepsilon,
\end{aligned}
$$

Thus $\left(z_{n}\right)_{n=1}^{N} \subset \Delta_{\varepsilon}(x)$ and as $\alpha>0$ is arbitrarily chosen, we are done.

\section{REFERENCES}

[1] T. A. Abrahamsen, Linear extensions, almost isometries, and diameter two, Extracta Math. 30 (2015), no. 2, 135-151.

[2] T. A. Abrahamsen, P. Hájek, O. Nygaard, J. Talponen, and S. Troyanski Diameter 2 properties and convexity, Studia Math. 232 (2016), no. 3, 227-242.

[3] T. A. Abrahamsen, J. Langemets, V. Lima, and O. Nygaard, On thickness and thinness of Banach spaces, Houston J. Math. 41 (2015), no. 1, 97-111.

[4] T. A. Abrahamsen, V. Lima, and O. Nygaard, Remarks on diameter 2 properties, J. Convex Anal. 20 (2013), no. 2, 439-452. MR 3098474

[5] _ Almost isometric ideals in Banach spaces, Glasgow Math. J. 56 (2014), no. 2, 395-407. MR 3187906

[6] J. Becerra Guerrero, G. López-Pérez, and A. Rueda Zoca, Subspaces of Banach spaces with big slices, Banach J. Math. Anal. 10 (2016), no. 4, 771-782. MR 3548625

[7] E. Behrends, M-structure and the Banach-Stone theorem, Lecture Notes in Mathematics, vol. 736, Springer, Berlin, 1979. MR 547509

[8] M. M. Day, Strict convexity and smoothness of normed spaces, Trans. Amer. Math. Soc. 78, (1955), 516-528, MR 0067351

[9] S. J. Dilworth, M. Girardi, and J. Hagler, Dual Banach spaces which contain an isometric copy of $L_{1}$, Bull. Polish Acad. Sci. Math. 48 (2000), no. 1, 1-12. MR 1751149 (2001e:46016)

[10] J. Dixmier, Sur un théorème de Banach, Duke Math. J. 15 (1948), 1057-1071. MR 0027440

[11] M. Fabian, P. Habala, P. Hájek, V. Montesinos, and V. Zizler, Banach space theory, CMS Books in Mathematics/Ouvrages de Mathématiques de la SMC, Springer, New York, 2011, The basis for linear and nonlinear analysis. MR 2766381 (2012h:46001)

[12] G. Godefroy, Metric characterizations of first Baire class linear forms and octahedral norms, Studia Math. 95 (1989), no. 1, 1-15.

[13] R. Haller, J. Langemets, and M. Põldvere, On duality of diameter 2 properties. J. Convex Anal. 22 (2015), no. 2, 465-482.

[14] P. Harmand, D. Werner, and W. Werner, M-Ideals in Banach Spaces and Banach Algebras, Lecture Notes in Math. 1547, Springer, Berlin-Heidelberg-New York, 1993. 
[15] Y. Ivakhno and V. Kadets, Unconditional sums of spaces with bad projections, Visn. Khark. Univ., Ser. Mat. Prykl. Mat. Mekh., 645 (2004), no. 54, 30-35.

[16] W. B. Johnson and J. Lindenstrauss, Basic concepts in the geometry of Banach spaces, In Handbook of the Geometry of Banach spaces, Elsevier (2001), Vol. 1, 1-84.

[17] V. M. Kadets, R. V. Shvidkoy, G. G. Sirotkin, and D. Werner, Banach spaces with the Daugavet property, Trans. Amer. Math. Soc. 352 (2000), no. 2, 855-873. MR 1621757 (2000c:46023)

[18] V. D. Milman, Geometric theory of Banach spaces. II. Geometry of the unit ball, Russian Math. Surveys 26 (1971), no. 6, 79-163.

[19] H. Pfitzner, A note on asymptotically isometric copies of $\ell_{1}$ and $c_{0}$, Proc. Amer. Math. Soc. 129 (2001), no. 5, 1367-1373. MR 1814162 (2001m:46008)

[20] R. V. Shvydkoy, Geometric aspects of the Daugavet property, J. Funct. Anal. 176 (2000), no. 2, 198-212. MR 1784413 (2001h:46019)

[21] M. A. Smith, Rotundity and smoothness in conjugate spaces, Proc. Amer. Math. Soc. 61 (1976), no. 2, 232-234 (1977). MR 0435807

[22] _ A curious generalization of local uniform rotundity, Comment. Math. Univ. Carolin. 25 (1984), no. 4, 659-665. MR 782015

[23] F. Sullivan, Geometrical peoperties determined by the higher duals of a Banach space, Illinois J. Math. 21 (1977), no. 2, 315-331. MR 0458124

[24] D. Werner, Recent progress on the Daugavet property, Irish Math. Soc. Bull. (2001), no. 46, 77-97. MR 1856978 (2002i:46014)

(T. A. Abrahamsen) Department of Mathematics, University of Agder, Postboks 422, 4604 KRISTIANSAND, NORWAY.

E-mail address: trond.a.abrahamsen@uia.no

URL: http://home.uia.no/trondaa/index.php3

(V. Lima) NTnu, Norwegian University of Science and Technology, Aalesund, Postboks 1517, N-6025 Ålesund Norway.

E-mail address: Vegard.Lima@ntnu.no

(O. Nygaard) Department of Mathematics, University of Agder, Postboks 422, 4604 KRISTIANSAND, NORWAY

E-mail address: Olav.Nygaard@uia.no

URL: http://home.hia.no/ olavn/

(S. Troyanski) Institute of Mathematics and Informatics, Bulgarian Academy of Science, Bl.8, acad. G. Bonchev str. 1113 Sofia, Bulgaria and Departamento de Matemáticas, Universidad de Murcia, Campus de Espinardo, 30100 Espinardo (MurCIA), SPAIN

E-mail address: stroya@um.es 